BULl. AUSTRAL. MATH. SOC.

10A35, 14G20, 30D35, 30G05.

VOL. 29 (1984), 109-117.

\title{
P-ADIC METHODS IN THE STUDY OF \\ TAYLOR COEFFICIENTS OF RATIONAL FUNCTIONS *
}

\author{
A. J. VAN DER POORTEN
}

\begin{abstract}
I sketch the proof of the so-called Hadamard Quotient Theorem: If the Hadamard quotient of two rational functions is a Taylor series with integer coefficients (more generally: with coefficients in some finitely generated subring of a field) then it is a rational function.
\end{abstract}

\section{Introduction}

Let $\mathbb{K}$ be a field of characteristic zero, and $r, s$ polynomials defined over $\mathbb{K}$ with $s(0) \neq 0$. A rational function is a quotient $r(X) / s(X)$ and we have a Taylor expansion

$$
\frac{r(X)}{s(X)}=\sum_{h \geq 0} a_{h} X^{h}
$$

We lose no generality in setting

$$
s(X)=1-s_{1} X-\cdots-s_{n} X^{n}=\prod_{i=1}^{m}\left(1-\alpha_{i} X\right)^{n(i)}
$$

where the $\alpha_{i}$ are distinct elements of $\mathbb{K}$. It is then easy to see that

Received 12 September 1983.

* This is a lecture delivered at the Special Meeting of the Australian Mathematical Society "Celebration of the 80th Birthday of Kurt Mahler", Canberra, July 26, 1983.

Copyright clearance Centre, Inc. Serial-fee code: 0004-9727/84 $\$ A 2.00+0.00$ 
we have

$$
a_{h+n}=s_{1} a_{h+n-1}+\ldots+s_{n} a_{h},
$$

so $\left(a_{h}\right)$ is a so-called recurrence sequence of order $n$, and, by expanding in partial fractions,

$$
a_{h}=\sum_{i=1}^{m} A_{i}(h) \alpha_{i}^{h}
$$

for polynomials $A_{i}$ of degree respectively $n_{i}-1$. These assertions hold for integers $h \geq \max (0, \operatorname{deg} r-n+1)$. The various descriptions of $\left(a_{h}\right)$ are equivalent.

\section{2. p-adification}

To study the sequence $\left(a_{h}\right)$ it is convenient to have $h$ a continuous parameter. Cassels [2] shows there are infinitely many rational primes $P$ so that the field $\mathbb{K}$ may be embedded in the field $Q_{p}$ of p-adic rationals whilst for each $\alpha=\alpha_{i}$

$$
\alpha^{p-1} \equiv 1(\bmod p) \text {, }
$$

equivalently:

$$
\operatorname{ord}_{p}\left(\alpha^{p-1}-1\right) \geq 1
$$

Then

$$
\log \alpha^{p-1}=\log \left(1-\left(1-\alpha^{p-1}\right)\right)
$$

is well-defined p-adically, and $\alpha^{t(p-1)}$ is given by the power series

$$
\exp \left(t \log \alpha^{p-1}\right)
$$

which converges for $t$ with ord $t>-1+1 / p-1$. Thus a recurrence sequence $\left(a_{h}\right)$ p-adifies to yield $p-1$ p-adic power series:

$$
a_{p, p}(t)=a(t(p-1)+r)=\sum_{i=1}^{m} A_{i}(t(p-1)+r) \alpha_{i}^{r} \exp \left(t \log \alpha_{i}^{p-1}\right), 0 \leq r<p-1 .
$$


It is now easy to prove the wonderful Skolem-Mahlem-Lech-Mahler Theorem: If $\left\{h: a_{h}=0\right\}$ is infinite then it is a union of finitely many arithmetic progressions (and finitely many isolated points). Indeed if the set is infinite then for some $r$ the p-adic analytic function $a_{p, r}$ vanishes infinitely often on the compact set $\mathbb{Z}_{p}$. Hence $a_{p, r}$ vanishes identically, so $a(h(p-1)+r)=0$ for $h$ in $\mathbb{Z}$. We proceed similarly for each remaining $a_{p, r^{*}}$ It is interesting to notice that in the situation just described it follows from a theorem of Ritt that

$$
\sin \frac{\pi(z-r)}{p-1} \mid \sum A_{i}(z) \exp \left(z \log \alpha_{i}\right)
$$

in the ring of exponential polynomials. Thus the Lech-Mahler theorem implies that an exponential polynomial with infinitely many integer zeros is sinful.

Some technical remarks. It can be shown by means of specialisation (see [5]) that here and in the sequel there is no loss of generality nor any introduced degeneracy in supposing $\mathbb{K}$ to be an algebraic number field of degree $d$, say, over $Q$. In that case the embedding into $Q_{p}$ is successful for primes $p$ of a set $P$ satisfying

$$
\prod_{\substack{p \leq x \\ p \in P}} p^{\frac{1}{p-1}} \geq C x^{1 / d} \text { with } c>0
$$

A major benefit of p-adification is obtained from the following fact: If $g(t)=\left[x_{h} t^{h}\right.$ converges for ord $t>-s$, say, and $\Delta g(i)=: g(i+1)-g(i)$ then

$$
\underline{\lim _{k} k^{-1} \text { ord } p^{\Delta^{k}} g(0) \geq s+1 / p-1} \text {. }
$$

Indeed we are given $\underline{\lim } h^{-1}$ ord $p_{h} \geq s$. But

$$
\Delta^{k} f(t)=\sum_{h \geq k} x_{h} \Delta^{k} t^{h} \quad \text { and }\left.\quad \operatorname{ord}_{p} \Delta^{k} t^{h}\right|_{t=0} \approx k / p-1
$$


We use this basic result as follows: it is not terribly difficult to see that $\left(a_{h}\right)$ is a recurrence sequence if and only if its Hankel-Kronecker determinants

$$
K_{h} a:=\left|a_{i+j}\right|_{0 \leq i, j<h}
$$

vanish for all sufficiently large $h$. But from elementary row and column operations one sees that

$$
K_{h} a=\left|\Delta^{k} a_{\bar{o}(p-1)+r}\right|_{i+j=k(p-1)+r} .
$$

So if $a_{t(p-1)+p}$ is given by p-adic power series converging beyond the p-adic unit disc we can show that $K_{h}$ a is divisible by a high power of $p$ (that is, is of high p-adic order).

\section{Hadamard division}

If $\sum a_{h} X^{h}, \sum b_{h} X^{h}$ are rational then their 'child's product' $\sum\left(a_{h} b_{h}\right) x^{h}$ is again rational; for $\left(a_{h}\right),\left(b_{h}\right)$ are given by generalised power sums whence so is $\left(c_{h}\right), c_{h}=a_{h} b_{h}$. The product above is frequently spoken of as the hadamard product of the power series. We wish to consider the hadamard quotient of rational functions. Polya [4] showed that if $\sum h a_{h} x^{h}$ is rational, and the $a_{h}$ all are integers (more generally, elements of a finitely generated subring $R$ of $\mathbb{K}$ ) then $\left[a_{h} X^{h}\right.$ is rational. Ultimately we have the theorem of Polya-Cantor [3] : if $f$ is a polynomial and $\sum f(h) a_{h} X^{h}$ is rational with the $a_{h}$ all in $R$, then $\sum a_{h} x^{h}$ is rational. The integrality condition is necessary: $\left[(h+1)^{-1} X^{h+1}\right.$ is not rational, but it is the hadamard quotient of $\left[x^{h+1}=X(1-X)^{-1}\right.$ by $\sum(h+1) x^{h+1}=X(1-X)^{-2}$.

We sketch a proof of the:

HADAMARD QUOTIENT THEOREM. Suppose $\left[c_{h} X^{h}, \sum b_{h} X^{h}\right.$ are Tayzor expansions of functions rational over $\mathbb{K}$ and that there is a sequence 
$\left(a_{h}^{\prime}\right)$ of elements of $R$ so that $a_{h}^{\prime} b_{h}=c_{h}, h \geq 0$. Then there is a rational function $\left[a_{h} X^{h}\right.$ with $a_{h} b_{h}=c_{h}, h \geq 0$.

Proof. We p-adify and consider quotients

$$
a(t(p-1)+r)=c(t(p-1)+r) / b(t(p-1)+r)
$$

of p-adic exponential polynomials. If $b$ is of order $n$ then $b$ has at most $n$ zeros in the disc with ord $t>-1 / n$. Hence for each $r: 0 \leq r<p-1$ there is a polynomial $f_{p, r}$ of degree at most $n$ so that $f_{p, r}(t(p-1)+r) a(t(p-1)+r)$ converges for $t$ with ord $p t \geq-1 / n$. The same holds for $f_{p}(t(p-1)+r) a(t(p-1)+r)$ if $f_{p}$ is the lowest common multiple of the $f_{p, r}$; we note that $f_{p}$ has degree at most $n(p-1)$.

Consider

$$
K_{H}\left(f_{p} a\right)=\left|f_{p}(i+j) a(i+j)\right|_{0 \leq i, j<H}
$$

We have shown that

$$
\lim ^{-2} \text { ord }_{p} K_{H}\left(f_{p} a\right) \geq\left(\frac{1}{n}+\frac{1}{p-1}\right) / p-1
$$

so, say with $H$ sufficiently large:

$$
\operatorname{ord}_{p} K_{H}\left(f_{p} a\right)>\frac{H^{2}}{n(p-1)} \text {. }
$$

But to obtain this result we use only that

$$
\operatorname{ord}_{p} \Delta^{k} f_{p}(\bar{o}(p-1)+r) a(\bar{o}(p-1)+r) \geq k\left(\frac{1}{n}+\frac{1}{p-1}\right) / p-1
$$

with $k(p-1)+r \leq 2 H$. Thus if we were to have truncated the coefficients of the $f_{p}$ modulo

$$
M(p ; H)=p^{2 H\left(\frac{1}{n}+\frac{1}{p-1}\right) /(p-1)^{2}}
$$

we would obtain the desired inequality for $\operatorname{ord}_{p} K_{H}\left(f_{p} a\right)$. 
However once coefficients are so truncated the $f_{p}$ become elements of $\mathbb{Z}[X]$ with coefficients not exceeding $M(p ; H)$. By the Chinese Remainder Theorem we may construct a polynomial $f$ in $\mathbb{Z}[X]$ with coefficients not exceeding

$$
M:=M(H)=\prod_{p \in P} M(p ; H)
$$

so that $f$ plays the role of $f_{p}$, each $p \in P$. The degree of $f$ is at most

$$
\max _{p \in p} n(p-1)
$$

To avoid the somewhat clumsy and naive notion of 'truncation' we can equivalently describe $f$ as being so constructed as to satisfy

$$
\left\|f-f_{p}\right\|_{p} \leq M(p ; H)^{-1},
$$

$p \in P$, thereby transforming our appeal to the Chinese Remainder Theorem to an appeal to the approximation theorem; here \|\|$_{p}$ is the valuation of the maximum of the coefficients.

As constructed, the polynomial $f$ has coefficients that are far too large and degree that is uneconomically small. Fortunately the following is plain: if $f_{0}$ be any non-zero polynomial in $\mathbb{Z}[X]$ then the remarks above, to wit that

$$
\operatorname{ord}_{p} K_{h}(f a)>h^{2} / n(p-1), H_{0}<h \leq H, p \in P
$$

remain true with $f$ replaced by $F=f_{0} f$. Accordingly we now appeal to the box-principle to choose $f_{0}$ so as to obtain $F$ with reasonably small coefficients and degree not too large. To see that we may replace $f$ by $F$ we need only recall that each $f_{p}$ might have been replaced by a multiple of itself.

Select $f_{O}$ of degree $N=, c_{1} H^{\frac{1}{2}}(\log H)^{-\frac{1}{2}}$. Here and in the immediate sequel $c_{0}, c_{1}, \ldots$ denote positive constants and $\mathrm{H}$ is supposed large with respect to the prevailing parameters $n$ and $p \in P$. Modulo $M$ there are 
some $M^{N}$ possibilities to choose $f_{0^{\circ}}$. We want $F=f_{O^{f}}$ to have coefficients no larger in absolute value than ${ } f_{0} / N$, modulo $M$ of course. Accordingly our 'boxes' each contain polynomials $F$ with coefficients differing modulo $M$ by no more than $\mathcal{F o}^{\circ}$. With $c_{0}$ appropriately large (not depending on $H$ ) there are fewer than $M^{N}$ such boxes. Hence our construction succeeds and we have

$$
\operatorname{ord}_{p} K_{h}(F a)>h^{2} / n(p-1) \quad H_{0}<h \leq H, p \in P
$$

with $F$ of degree $c H^{\frac{1}{2}}(\log H)^{-\frac{1}{2}}$ and with coefficients not exceeding $M_{0} / N$ in absolute value. A priori $H_{O}$ need only be large enough to validate the p-adic inequalities. It certainly suffices to set $H_{0}=H^{\frac{1}{2}} \log H$.

We now need a suitable archimedean upper bound for the algebraic numbers $K_{h}(\mathrm{Fa})$.

Technical remark. The correct measure of the size of a sequence of algebraic numbers is provided by Bombieri [1] p. 37 in his discussion of G-functions. Since the $a_{h}$ belong to a finitely generated subring $R$ of $K$ the sequence $\left(a_{h}\right)$ has finite size $\rho$, say. For us it is convenient to define

$$
\sigma_{h}(a)=\sum_{v} \max _{j \leq h}\left|a_{j}\right|_{v}
$$

with the sum the appropriately normalised valuations $v$ of $\mathbb{K}$. Then

$$
\sigma(a)=\overline{\lim }_{h \rightarrow \infty} h^{-1} \log \sigma_{h}(a)=\log \rho .
$$

It then follows that

$$
\overline{\lim }_{h \rightarrow \infty} h^{-2} \log \sigma_{h}(K(a))=\log \rho .
$$

Then with the $F$ chosen as above we obtain

$$
\log \sigma_{h}(K(F a))<h^{2} \log \rho+c_{2} h H^{\frac{1}{2}}(\log H)^{\frac{1}{2}}
$$

But for $H_{0}<h \leq H$ and $p \in P$ 


$$
\operatorname{ord}_{p} K_{h}(F a)>h^{2} / n(p-1)
$$

Hence if $H_{0}=H^{\frac{1}{2}} \log H$ then for $H_{0}<h<H$ we have

$$
h^{-2} \log \sigma_{h}(K(F a))<\log \rho+c_{3}(\log H)^{-\frac{1}{2}}
$$

But by the product formula $K_{h}(F a)$ vanishes if

$$
\sum_{p}(\log p) \operatorname{ord}_{p} K_{h}(F a)>d \log \sigma_{h}(K(F a)) .
$$

Indeed it is this formula that justifies the measure $\sigma_{h}$ we have introduced above.

We can now return to the main argument. From the remarks above we see that $K_{h}(F a)=0$ for $H^{\frac{1}{2}} \log H<h<H$ if

$$
\sum_{p \in P} \frac{\log p}{n(p-1)}>d \log \rho+d c_{3}(\log H)^{-\frac{1}{2}}
$$

We note that though $c_{3}$ depends on $P$ it remains bounded if $P$ grows, hence since the sum over $p \in P$ is unbounded as $P$ grows we can certainly achieve the condition above for a suitably large choice of the set $P$.

The vanishing of $K_{h}(F a)$ for $H^{\frac{1}{2}} \log H<h<H \quad$ implies readily that there is a recurrence sequence $\left(d_{h}\right)$ of order at most $H^{\frac{1}{2}} \log H$ so that

$$
d_{h}=F(h) a_{h}=F(h) c_{h} / b_{h}
$$

for $H^{\frac{1}{2}} \log H<h<H$. But the recurrence sequences $\left(b_{h} d_{h}\right)$ and $\left(F(h) c_{h}\right)$ then coincide over a range considerably greater than is their order. Hence they coincide for all $h$. It follows that $F$ divides $b d$ in the ring of exponential polynomials. By the Polya-Cantor lemma we lose no generality in assuming that $b$ has no polynomial factor; for any such factor must also be a factor of $c$ and so we may suppose it to have been removed. Hence $F$ divides $d$ in the ring of exponential polynomials, for the quotient satisfies the conditions of the Polya-Cantor lemma. Hence, as we wished to show, $\left(a_{h}\right)$ is indeed a recurrence sequence. To complete the proof we should deal with the case where some $b_{h}$ vanish and with the general case where $\left(a_{h}\right)$ is not defined over an 
algebraic number field. These technicalities seem inappropriate to this sumary.

\section{Concluding Remarks}

The p-adic methods employed above were either introduced or flowered in the work of Kurt Mahler. It seems especially appropriate to describe a recent application of his ideas on this the occasion of his 80th birthday.

\section{References}

[1] E. Bombieri, "On G-functions", in H. Halberstam and C. Hooley (eds.) Recent Progress in Analytic Number Theory (Academic Press, 2 , $1981,1-67)$.

[2] J.W.S. Casse1s, "An embedding theorem for fields", BulZ. Austral. Math. Soc. 14 (1976), 193-198. Addendum, ibid. 14 (1976), 479-480.

[3] D.G. Cantor, "On arithmetic properties of the Taylor series of rational functions II", Pacific J. Math. 41 (1972), 329-334.

[4] G. Polya, "Arithmetische Eigenschaften der Reihenentwicklungen rationaler Functionen", J. fïr die reine u. angew Math. 151 (1920), 1-31.

[5] A.J. van der Poorten and H.P. Schlickewei, "The growth conditions for recurrence sequences", Invent. Math., to appear.

School of Mathematics and Physics,

Macquarie University,

North Ryde,

New South Wales 2113,

Australia. 